\title{
Temporal variation and the evolution of a parasitoid foraging cue
}

\author{
Chad E. Brassil \\ C.E. Brassil (cbrassil2@unl.edu), Dept of Zoology, Univ. of Toronto, 25 Harbord St, Toronto, Ontario, Canada, M5S 3G5. Present \\ address: School of Biological Sciences, Univ. of Nebraska, Lincoln, NE 68588-0118, USA.
}

\begin{abstract}
This work details theory in which selection favors generalists in a more variable environment. Specifically, in a two-host-one-parasitoid model, temporal variation in host abundances alters the optimal searching strategy and leads to the evolution of more generalist parasitoid strategies. Consistent with empirical observations, parasitoids learn host/plant odors, and use them as a cue to search for oviposition sites. The amount of unsuccessful search time required before a parasitoid alters its searching cues (the "giving-up time") is modeled in order to understand the evolutionarily optimal giving-up times under a variety of conditions. When host abundances vary across time, a generalist parasitoid strategy evolves with short giving-up times as it is likely that the host initially favored by a parasitoid will now have a low abundance. In contrast, when populations reach stable dynamics across time, giving-up times typically evolve to longer times, i.e. parasitoids remain specialized longer. The effect of temporal fluctuations is consistent across variation caused by endogenous population interactions and, to some degree, by environmental stochasticity. The conclusions are robust in that there is a strong degree of concordance between the results of a stochastic, individual-based model and a deterministic, numerical model. As an extension, spatial variation in hosts that leads to unequal tradeoffs between generalist parasitoids and specialist parasitoids may also result in the evolution of reduced giving-up times.
\end{abstract}

Optimality models predict the evolutionary equilibrium of a trait given a set of ecological conditions. Analysis of optimality models commonly involves restricting parameters to situations in which a stable equilibrium exists. Only a few studies (e.g. Abrams 1997, Nuismer and Doebeli 2004) have examined the consequences of unstable dynamics for the evolution of traits. Here I examine the importance of temporal variation in population sizes on the evolution of a parasitoid foraging trait in order to address the following questions. Is the effect of population cycles on interspecific interactions similar across all ecological parameters? Can both positive and negative indirect interactions occur in stable systems, or are there some interactions that can only be observed in the presence of temporal variation? Can both endogenous population cycles and exogenous environmental variation lead to a similar evolutionary response in the trait? These broad ecological questions are addressed in the context of a specific ecological system in which two host populations are attacked by a single parasitoid population.
The trait of interest is a behavioral rule used by parasitoids to make decisions when searching for hosts. Learning environmental cues associated with the presence of suitable hosts is an important part of the strategy used by parasitoids to search for hosts on which to lay eggs. Parasitoids can learn both host and plant odors as a means of locating hosts (Turlings et al. 1993). The earliest learning can take place upon emergence from a host when the parasitoid experiences plant and host fecal chemicals and develops a natal host preference based on these early experiences (Kester and Barbosa 1991). Natal host preference can be reinforced by positive oviposition experience (Vet and Groenewold 1990). Importantly, there is also evidence that parasitoids forget odor cues. In the above laboratory experiments, parasitoids forget foraging cues when they fail to find a host after a certain amount of time using that cue. This contrasts with previous work that has modeled learning as a fixed preference throughout the season (Hastings and Godfray 1999). There appears to be a sensitive learning period early in the lifespan of 
the adult parasitoid (Sheehan and Shelton 1989, Vet and Groenewold 1990, Kester and Barbosa 1991); however, most parasitoids continue to modify foraging cues later in life (Kerguelen and Carde 1997).

The giving-up time (GUT) models traditionally used to model patch selection (Van Alphen and Galis 1983) can be used to model the use of odor cues by parasitoids because searching for hosts is analogous to searching for food. Instead of decisions about departing from patches, parasitoids make decisions about givingup on the use of a species-specific searching cue to find a host. This represents a simple model of learning that is consistent with many empirical studies of parasitoid foraging (Sheehan and Shelton 1989, Papaj and Vet 1990, Kester and Barbosa 1991, Grasswitz and Paine 1993a, 1993b).

Although the optimal search strategy for patchy resources is often not precisely described by a GUT model (Green 1984), previous theory based on patch selection has found that a GUT strategy can be nearly optimal depending on the resource distribution (Waage 1979, Iwasa et al. 1981, McNair 1982, Green 1984, Driessen et al. 1995). Presumably, GUT models are only caricatures of the actual mechanisms used by parasitoids. Nevertheless, it is important to understand how evolution acts in such models because they approximate a mechanism that can be easily implemented, which may not be the case for the strict optimal strategy.

The optimal GUTs were determined using two approaches: (1) a deterministic model based on the expected values from a stochastic encounter process; and (2) an individual-based model with stochastic hostparasitoid encounters. For model parameters that lead to relatively stable population dynamics, the giving-up time of the parasitoids evolves to moderate or long times. The presence of population cycles, generated by feedback loops in the population dynamics, or by environmental variation, leads to shorter giving-up times.

\section{Model}

The model includes two host populations, $\mathrm{H}_{\alpha}$ and $\mathrm{H}_{\beta}$, and a shared parasitoid, P. Each host population utilizes different resources and could be distinguished by parasitoids using cues from the host, the plant, or the host-plant complex. When parasitoids search for oviposition sites, they adopt either a specialist or a generalist strategy. In real systems, searching strategies are probably characterized by a continuous variable with parasitoids utilizing a Bayesian decision process that accounts for multiple encounter events. A simplified dichotomous approach is adopted here for the purpose of creating a tractable model. When a generalist parasitoid encounters a host, it learns hostspecific cues and switches to a specialist strategy. Specialist parasitoids learn their first cues at emergence and became specialists on their natal host (Kester and Barbosa 1991). Specialist parasitoids $P_{i}$ have an attack rate $a$ on host $i$ and an attack rate 0 on the other host. If a parasitoid fails to encounter a host after giving-up time $\mathrm{g}$, then the parasitoid switches to a generalist strategy with an attack rate $\mathrm{z} \times$ a on both hosts, where $\mathrm{z}$ (between 0 and 1) represents the reduction in attack rate on a host as compared to the specialist attack rate. There was an equitable tradeoff in attack rates such that $\mathrm{z}=0.5$ for most simulations examined here.

The current model differs from previous probabilistic models that assume giving-up time is very small relative to the total length of the season (Iwasa et al. 1981). Here giving-up time is free to take on any value and is explicitly measured relative to the length of the season, i.e. the length of the available time that both the hosts and parasitoids are alive and able to interact.

First, I develop a simplified analytical model in which all parasitoids have an identical giving-up time for host-specific searching. The analytical model illustrates within-season dynamics, but it has limitations. Solutions of the analytical model require complex numerical algorithms, and the model involves assumptions about genotype distribution and the strength of selection. Therefore, I also develop an individual-based model in which each parasitoid has a unique genotype for giving-up time. The individual-based model relaxes assumptions about genotype distribution and weak selection made by the analytical model.

\section{Analytical model}

For the analytical model, parasitoids begin adult life as specialists on their natal host. From time 0 to g, the probability of an $\alpha$-specialist parasitoid having no host encounters is $\exp \left(-\mathrm{a} \mathrm{H}_{\alpha} \mathrm{g}\right)$, derived from

$\frac{\mathrm{dP}_{\alpha, 0}}{\mathrm{dt}}=-\mathrm{aP}_{\alpha, 0} \mathrm{H}_{\alpha}$

where $P_{\alpha, 0}$ are $\alpha$-specialist parasitoids that have had no host encounters and parasitoids encounter hosts at rate a. An equivalent equation models the $\beta$-specialist parasitoids. At time $\mathrm{g}$, the number of specialist parasitoids on host $\alpha$ drops instantaneously from $\mathrm{P}_{\alpha}$ to $\mathrm{P}_{\alpha}\left(1-\exp \left(-\mathrm{a} \mathrm{H}_{\alpha} \mathrm{g}\right)\right)$ as those parasitoids that have had no contact with a host switch to a generalist strategy (Fig. 1). In this model, an encounter with either a parasitized or non-parasitized host is sufficient for the parasitoid to remain a specialist. This describes parasitoids that cannot discriminate between parasitized and non-parasitized hosts, or parasitoids that, despite the ability to discriminate, continue to use search cues after 


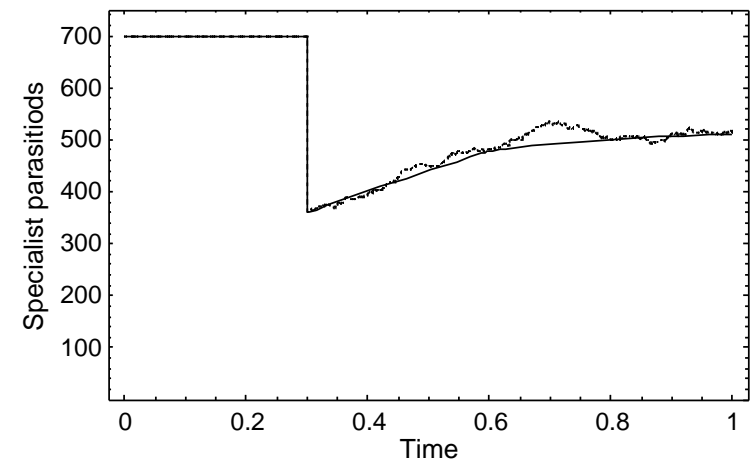

time

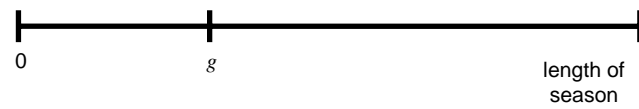

Fig. 1. Example of within-season parasitoid dynamics in which the number of parasitoids that are specialized on $\beta$ drops at the giving-up time $(\mathrm{g})$ and then increases as parasitoids switch from a generalist strategy back to a specialist strategy. The solid line is the expected specialist population based on Eq. 2. The dashed line is a single run of the individual-based model with an identical genotype $\mathrm{g}$ for all parasitoids. $\left(\mathrm{P}_{\alpha, 0}=1000, \mathrm{P}_{\beta, 0}=700, \mathrm{H}_{\alpha}=1000, \mathrm{H}_{\beta}=2000\right.$, $\mathrm{a}=0.0012, \mathrm{z}=0.5, \mathrm{~g}=0.3$ )

encountering previously parasitized hosts. Parasitoids may continue to use these cues either because of opportunities for superparasitism (not modeled here) or because previously parasitized hosts may indicate the presence of non-parasitized hosts.

Following the initial giving-up time g, the number of $\alpha$-specialist parasitoids continues to change as a function of time $\mathrm{P}_{\alpha}(\mathrm{t})$. Those specialist and generalist parasitoids that encountered an $\alpha$-host g time units ago (in the square brackets of Eq. 2) and have not encountered a host since that time, the fraction $\exp \left(-\mathrm{aH}_{\alpha \mathrm{g}}\right)$, will switch to a generalist strategy. Generalist parasitoids immediately switch back to, or learn, a specialist strategy when they encounter a host (positive term of Eq. 2),

$$
\begin{aligned}
\frac{\mathrm{dP}}{\mathrm{dt}}= & -\exp \left(-\mathrm{aH}_{\alpha} \mathrm{g}\right)\left[\mathrm{aH}_{\alpha} \mathrm{P}_{\alpha}(\mathrm{t}-\mathrm{g})\right. \\
& \left.-\mathrm{zaH}_{\alpha}\left(\mathrm{P}-\mathrm{P}_{\alpha}(\mathrm{t}-\mathrm{g})-\mathrm{P}_{\beta}(\mathrm{t}-\mathrm{g})\right)\right] \\
& +\mathrm{zaH}_{\alpha}\left(\mathrm{P}-\mathrm{P}_{\alpha}(\mathrm{t})-\mathrm{P}_{\beta}(\mathrm{t})\right)
\end{aligned}
$$

An equivalent equation models the $\beta$-specialist parasitoids. The solution to this differential equation consists of multiple segments, each $\mathrm{g}$ time units long (Fig. 1). An analytical solution of Eq. 2 for $\mathrm{P}_{\alpha}(\mathrm{t})$ and $\mathrm{P}_{\beta}(\mathrm{t})$ is sufficiently complicated to be of little use. Analytical insights are not evident even when the multi-segment aspect of the solution is approximated as a smooth function after the first segment because the complexity that arises from $\mathrm{dP}_{\alpha} / \mathrm{dt}$ being dependent on both specialist densities $\mathrm{P}_{\alpha}(\mathrm{t})$ and $\mathrm{P}_{\beta}(\mathrm{t})$. However, a solution can be found using numerical methods.

Within-season foraging dynamics are modeled in continuous time while across-season population dynamics are modeled in discrete time. For both the deterministic and individual-based model, across-season system dynamics follow a host-parasitoid model with density-dependence (Beddington et al. 1975),

$$
\begin{aligned}
& \mathrm{H}_{\alpha}(\mathrm{t}+1)=\mathrm{f}_{\alpha} \mathrm{H}_{\alpha}(\mathrm{t}) \exp \left(\mathrm{r}_{\alpha}\left(1-\frac{\mathrm{H}_{\alpha}(\mathrm{t})}{\mathrm{k}_{\alpha}}\right)\right) \\
& \mathrm{H}_{\beta}(\mathrm{t}+1)=\mathrm{f}_{\beta} \mathrm{H}_{\beta}(\mathrm{t}) \exp \left(\mathrm{r}_{\beta}\left(1-\frac{\mathrm{H}_{\beta}(\mathrm{t})}{\mathrm{k}_{\beta}}\right)\right) \\
& \mathrm{P}(\mathrm{t}+1)=\left(1-\mathrm{f}_{\alpha}\right) \mathrm{H}_{\alpha}(\mathrm{t})+\left(1-\mathrm{f}_{\beta}\right) \mathrm{H}_{\beta}(\mathrm{t})
\end{aligned}
$$

with growth rate $r_{i}$ and carrying capacity $k_{i}$ for host $i$. The fraction of the host population $i$ that escapes attack by a parasitoid is $f_{i}$ and is determined by encounters between hosts and parasitoids. In the individual-based simulation, $f_{i}$ varies due to the stochastic process of encounters, so the deterministic model is used to examine the dynamic stability of Eq. 3 in the absence of individual variation and stochasticity.

The parameter $\mathrm{g}$ was allowed to evolve across generations based on the assumptions of a quantitative genetics model, which implicitly assumes weak selection and a distribution of trait values that is narrow relative to the width of the fitness function (Abrams et al. 1993). The fitness $\mathrm{W}(\mathrm{g}, \overline{\mathrm{g}})$ of an individual with a giving-up time $\mathrm{g}$ in a population with a mean giving-up time of $\bar{g}$ equals the expected number of nonparasitized hosts attacked by that individual over the course of the season. This is a function of the mean per capita attack rate on each host with a giving-up time $g$ and the number of non-parasitized hosts of type $i$ in a population of parasitoids that have a mean giving up time $\overline{\mathrm{g}}, \mathrm{H}_{\mathrm{i}, 0}(\overline{\mathrm{g}}, \mathrm{t})$.

$$
\begin{aligned}
& \mathrm{W}(\mathrm{g}, \overline{\mathrm{g}})= \\
& \int_{0}^{1} \frac{\mathrm{aP}_{\alpha}(\mathrm{g}, \mathrm{t})+\mathrm{za}\left(\mathrm{P}-\mathrm{P}_{\alpha}(\mathrm{g}, \mathrm{t})-\mathrm{P}_{\beta}(\mathrm{g}, \mathrm{t})\right)}{\mathrm{P}} \mathrm{H}_{\alpha, 0}(\overline{\mathrm{g}}, \mathrm{t}) \\
& +\frac{\mathrm{aP} \mathrm{P}_{\beta}(\mathrm{g}, \mathrm{t})+\mathrm{za}\left(\mathrm{P}-\mathrm{P}_{\beta}(\mathrm{g}, \mathrm{t})-\mathrm{P}_{\beta}(\mathrm{g}, \mathrm{t})\right)}{\mathrm{P}} \mathrm{H}_{\beta, 0}(\overline{\mathrm{g}}, \mathrm{t}) \mathrm{dt}
\end{aligned}
$$

This assumes that the parasitoid that emerges from a host is a progeny of the first parasitoid to attack a host. The change in the $\mathrm{g}$ between generations is related to the genetic variance, $\mathrm{V}$, and the fitness gradient which was calculated numerically with $\Delta \mathrm{g}^{*}=10^{-7}$.

$\frac{\bar{g}_{\mathrm{t}+1}=\overline{\mathrm{g}}_{\mathrm{t}}+\mathrm{V} \Delta \mathrm{W}\left(\mathrm{g}^{*}, \overline{\mathrm{g}}_{\mathrm{t}}\right)}{\Delta \mathrm{g}^{*}}$ 
The equation that describes the evolution of $\mathrm{g}$ involves additional numerical integration, and the solution becomes so computationally intensive that it is equally efficient to simulate the system using an individualbased model (IBM). It is more difficult to characterize population dynamics in the individual-based simulation; however, the individual-based model can more easily include relevant biology such as a wider range of genotypes in the population, and it automatically incorporates the demographic stochasticity inherent in parasitoid-host encounters in a finite population. Both the IBM and the deterministic, numerical model were analyzed and compared in order to understand the evolutionarily optimal giving-up times under a variety of conditions.

\section{Individual-based model}

The individual-based model simulates stochastic encounters between individual hosts and parasitoids, and genes are individually tracked. Parasitoids make individual searching decisions based on their experiences and pass on the genes associated with their behavioral decisions. The giving-up time is coded by a string of 100 additive "binary" loci in a diploid genome resulting in a normalized phenotype g between 0 and 1. Mating is panmictic; mutation and recombination takes place as eggs are laid. Mutation was simulated by randomly switching the value of the gene at each of the 100 loci with a probability of 0.00008 per locus. This probability was chosen because it resulted in simulations for which mutation did not swamp out selection, yet some variation was maintained, and the value is within the range of realistic values (Griffiths et al. 1996). Host-parasitoid encounters and parasitoid switching behavior are simulated in continuous time by stochastically simulating the time until the next discrete event (Gillespie 1976). The individual-based model was written in $\mathrm{C}++$, using the Mersenne twister random generator (http://www.agner.org/random). Results were compared to a separate IBM written in Mathematica 5.0 and to numerical solutions of Eq. 2 in order to check for coding and mathematical errors.

Simulations were run for 5000 generations with mean population sizes and variance in population sizes calculated over the last 3000 generations. Parameters were varied both systematically and randomly to explore the range of model dynamics. Stable parameter space was examined by systematically changing a single host parameter, either the growth rate, $\mathrm{r}_{\beta}$, or the carrying capacity, $\mathrm{k}_{\beta}$. For each parameter set, simulations were repeated three times in order to examine variation among individual stochastic runs. Given that the unit of $\mathrm{k}$ is individuals per area, the IBM was run with an area of 2000 .

\section{Analysis}

\section{Stable parameter space}

At low attack rates, a, and low host growth rates, $r$, the deterministic model is stable due to host densitydependence. Even for these parameters, the individual-based model will have temporal variation in population sizes because of the stochastic process of host-parasitoid encounters. Temporal variance in the IBM is predicted to follow a beta binomial distribution, i.e. elevated variance as compared to a binomial distribution. Based on preliminary inspections, parameters that lead to variance ratios (observed: predicted based on binomial) less than 12 in the IBM will be considered stable, because in the absence of stochastic encounters, population sizes would reach a stable equilibrium in most such systems.

For stable parameters, the evolution of $\mathrm{g}$ can be explained by understanding the selective pressures on each parasitoid sub-population. An ideal parasitoid would focus solely on the most abundant host, foraging broadly when abundances are equal (Stephens and Krebs 1986). For parasitoids emerging from the abundant host an approximation to this ideal strategy can be accomplished within the constraints of the GUT model, whereas parasitoids emerging from the less abundant host would benefit by immediately foraging broadly. However, parasitoids from the abundant host drive the evolution of $g$ because of their larger numbers. The equilibrium giving-up time $\mathrm{g}$ can be explained by understanding the evolutionary pressure on parasitoids emerging from the dominant host and by examining the difference between the host densities and the total host density.

For parameters leading to a stable equilibrium, an increase in a growth parameter of a host (such as $r_{\alpha}$ or $\mathrm{k}_{\beta}$ ) decreases the equilibrium g. Intuitively, one may expect the evolution of large values of $\mathrm{g}$ when an increase in a growth parameter leads to a large difference between host densities, but that is not the case. Sufficiently large increases in the growth parameter result in $\mathrm{g}$ asymptotically approaching a minimum value (Fig. 2a for $k_{\beta}$ up to 1.9). The initial decrease in the equilibrium $g$ with an increase in a growth parameter of a host is due to an equalization of equilibrium host abundances as the growth rate of the least abundant host population increases (Fig. 2b). At equilibrium, the abundances of non-parasitized individuals of the two host populations reverse relative rank within a season. As the host densities become more similar at the beginning of the season, they cross over in rank abundance earlier in the season. Equilibrium giving-up time decreases because the most abundant parasitoids that are emerging from the most abundant hosts should become generalists sooner. However, there 

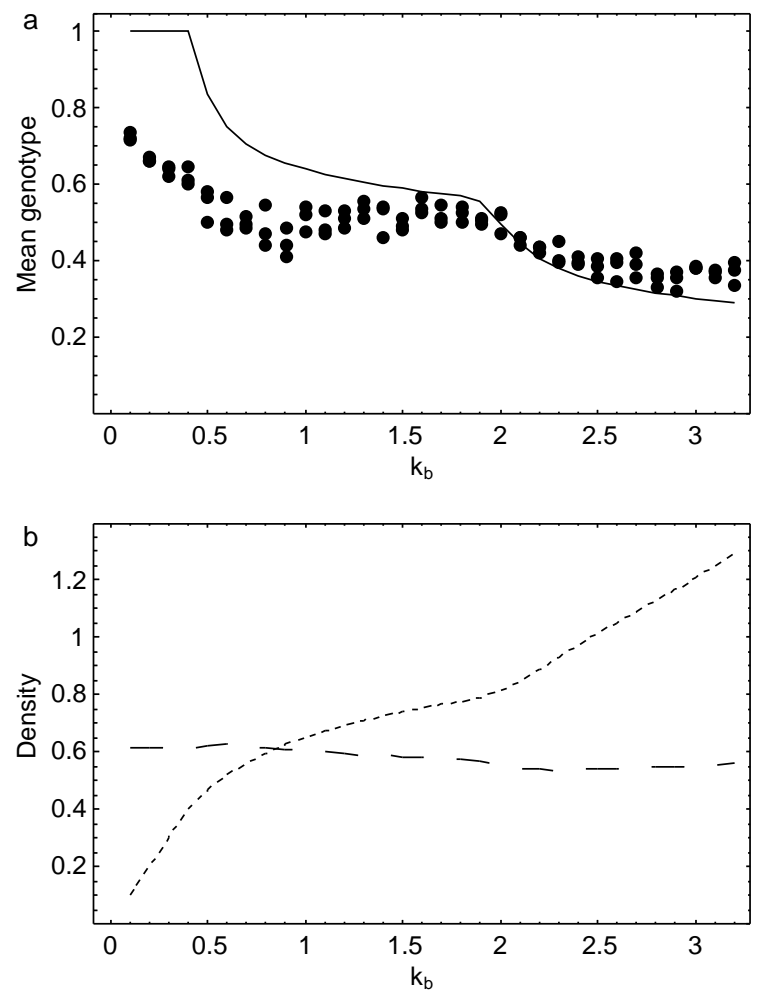

Fig. 2. Change in mean genotype with change in the carrying capacity of host $\beta$. In this case, population densities cycles when $\mathrm{k}_{\beta}>1.9$. (a) The solid line is the equilibrium giving-up time for the deterministic model. Each circle represents a run of the individual-based model. Three simulations are plotted for each value of $k_{\beta}$. (b) The mean density of each host population from the deterministic model. The long-dashed line represents $\mathrm{H}_{\alpha}$, the short-dashed line represent $\mathrm{H}_{\beta}$. $(\mathrm{a}=2$, $\left.\mathrm{r}_{\alpha}=1.1, \mathrm{r}_{\beta}=1.5, \mathrm{k}_{\alpha}=1, \mathrm{z}=0.5\right)$.

is no increase in the giving-up time with increases in the growth parameter of the abundant host despite an increasing difference between host densities (Fig. 2b). This is because larger host densities lead to larger parasitoid densities and these in turn cause a faster decline of non-parasitized hosts within a season. The faster decline leads to an earlier crossover in hostabundances than one would expect based on the same host ratio at smaller host densities. However, giving-up times below $\sim 0.4$ were never found for parameter space that produced stable equilibria. At equilibrium, the parasitoid abundances always correspond to host abundances in such a way that a crossover in host abundances never occurs early within the season.

The above analysis assumes each host has the same attack rate. Increasing the attack rate on the numerically dominant host (while maintaining the same average attack for both hosts) results in the evolution of relatively reduced giving-up times. The larger attack rate on the numerically dominant host leads to a reduction in the host density and an increase in the density of the dominant parasitoid. The increased density of parasitoids emerging from the dominant host and the increased attack rate on the dominant host more quickly depletes the dominant host within a season, and shorter giving-up times evolve. For example given the parameters in Fig. 2 , at $\mathrm{k}_{\beta}=1.3$, the givingup times evolved to 0.49 with attack rates of 1.4 and 2.6 on $\mathrm{H}_{\alpha}$ and $\mathrm{H}_{\beta}$ respectively as compared to 0.60 when both attack rates were 2.0.

A few realistic biological assumptions can be included in the IBM which are not included in the deterministic model. 1) Accounting for drift and mutation results in different evolutionary outcomes when selection becomes weak. Mutations in this model push the giving-up time genotype toward 0.5 because allelic values are constrained to be 0 or 1 , resulting in a mutational bias. As a consequence of the mutationselection balance, the actual mean genotypes in the individual-based model are closer to 0.5 than that predicted by the deterministic model (Fig. 2a). 2) Parasitoids that can make foraging decisions based on the parasitism state of the host do not substantially alter the dynamics and mean $\mathrm{g}$ as compared to those presented in Fig. 2.

\section{Unstable parameter space}

For many parameter values the equilibrium is unstable. The parasitoid population and host populations cycle deterministically when the attack rate, a, is sufficiently large and the amplitude of the cycles increases as the attack rate increases (Kaitala et al. 1999). Large amplitude cycles lead to the evolution of shorter giving-up times. This is illustrated in Fig. 2 by the reduction in giving-up time when $\mathrm{k}_{\beta}>1.9$, a parameter range for which population cycles persist. The pattern observed when exploring single parameters systematically is supported by analysis in which all parameters where chosen randomly. Combinations of random parameter values from the ranges $\mathrm{a} \in(4,1), \mathrm{r}_{\alpha} \in(3.5,1)$, $\mathrm{r}_{\beta} \in(3.5,1), \mathrm{k}_{\alpha} \in(3,0)$, and $\mathrm{k}_{\beta} \in(3,0)$ were chosen 500 times. A mean $g>0.4$ was never observed for stable cases and often observed for systems characterized by cycles (defined by a high variance ratio).

Deterministic population cycles can change the rank order of host population sizes among generations. The host with the larger population size often crashes before the host with the smaller population size, especially when host demographic parameters differ. At these times, a large population of parasitoids that emerged from a previously numerous host are in a generation with few natal hosts, but many non-natal hosts. These generations drive selection for shorter giving-up times. As a consequence, population cycles that are driven by a 
high host-parasitoid encounter rate result in the evolution of shorter giving-up times because the host-crash across generations increases the selection pressure for parasitoids to forage more broadly.

Environmental variation can lower the giving-up time in a manner similar to deterministic, endogenous cycles. In order to examine the ability of environmental variation to generate population cycles that affect the evolution of $\mathrm{g}$, the values of demographic parameters were modeled as dependent on an environmental variable drawn from a random distribution. Simulations were conducted in which the growth rates, $r$, for each host species were randomly chosen each generation. Results show that environmental variation leads to the evolution of lower giving-up times (Fig. 3). If host growth rates were negatively correlated between hosts, then giving-up times evolved to lower levels than if host growth rates were positively correlated. However, environmental variation did not reduce giving-up times
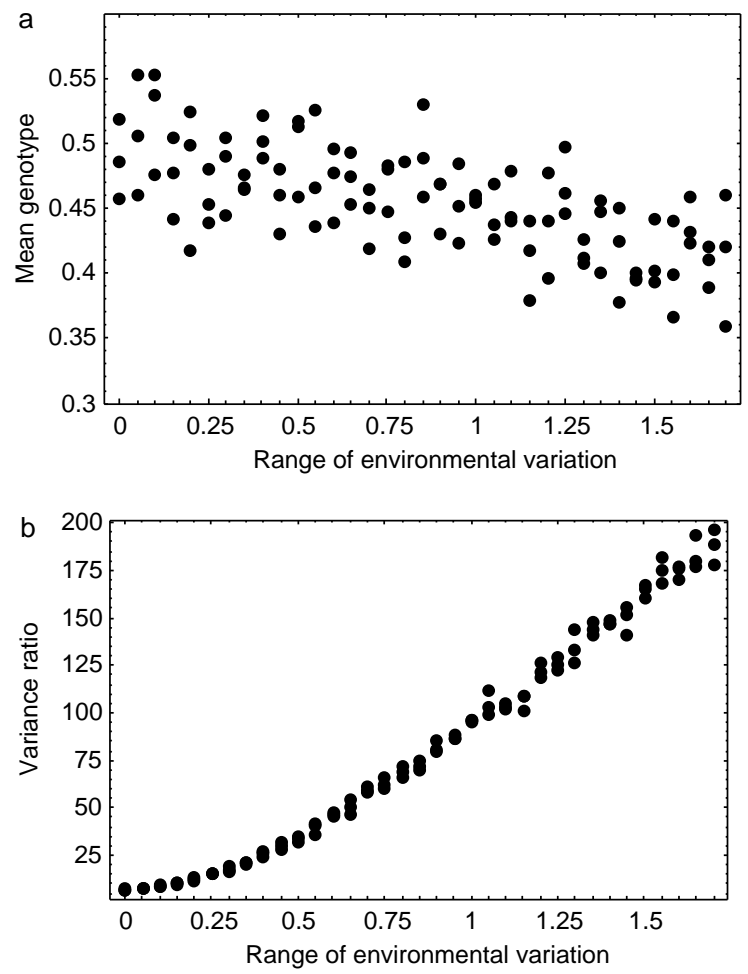

Fig. 3. (a) Response of giving-up time to increasing environmental variation. Growth rates are varied within a range for which the system is stable at fixed growth rate values. Growth rates for $\alpha$-hosts and $\beta$-hosts are randomly drawn from a uniform distribution equal to $1.8 \pm$ the range of environmental variation. (b) Variance rate, which is defined as the actual temporal variance of the parasitoid population divided by the variance expected if host-parasitoid encounters were determined by a pure binomial process. $(\mathrm{a}=2.23$, mean $\mathrm{r}_{\alpha}=1.8$, mean $\left.\mathrm{r}_{\beta}=1.8, \mathrm{k}_{\alpha}=1.23, \mathrm{k}_{\beta}=1.23, \mathrm{z}=0.5\right)$ to as low a value as found in some systems with endogenously driven cycles. The limited effect of environmental variation may be partially due to the restricted values over which parameters could vary. To ensure that fluctuations in population sizes were due to exogenous processes, the range of the demographic parameter was restricted to values for which the population dynamics would be stable.

\section{Alternative tradeoffs in attack rates}

The above analysis was restricted to $\mathrm{z}=0.5$ in order to understand the importance of temporal variation. However, the value of $\mathrm{z}$ is a major determinant of the evolution of giving-up times. When $\mathrm{z}=0.5$, there is a perfectly additive tradeoff such that the generalist and specialist strategies have the same search effort per host. This describes a situation in which searching for one host type precludes encounters with the other host type, for example if the two host populations occur in two separate patches and travel time between the patches is trivial. When $\mathrm{z}<0.5$, a generalist parasitoid is less efficient than a specialist (Fig. 4a). Parasitoid host location has traditionally been thought of as a hierarchy of cues from general habitat location, to microhabitat location, to individual host location (Godfray 1994). If generalist parasitoids abandon more specific cues in the hierarchy and rely on less specific cues, then generalist parasitoids may be less efficient overall (Steidle et al. 2001). Conversely, a generalist parasitoid could be more efficient if the hosts' habitats are interspersed and a generalist parasitoid spends less time passing over non-target habitat than a specialist parasitoid.

Changes in $\mathrm{z}$ can have an effect as large as or larger than that of the amplitude of cycling. At the extreme zvalues of 0 and 1 , the optimal parasitoid strategy is a giving-up time of 1 and 0 respectively. In the individual-based model, the mean $\mathrm{g}$ can evolve during stable dynamics to nearly the full range of all potential values along a gradient of $\mathrm{z}$. The mean $\mathrm{g}$ is only prevented from reaching the extremes by mutation pressure (Fig. 4b).

\section{Discussion}

Given the assumption of an additive trade-off in attack rates $(z=0.5)$, the part of parameter space where between-season population dynamics are stable is characterized by the relative rank abundance of nonparasitized hosts of the two types crossing over relatively late in the season. As a result, giving-up times evolve to values greater than 0.4 . Lower giving-up times evolve in two situations: 1) generalist parasitoids have only a minor reduction in searching efficiency on each species 

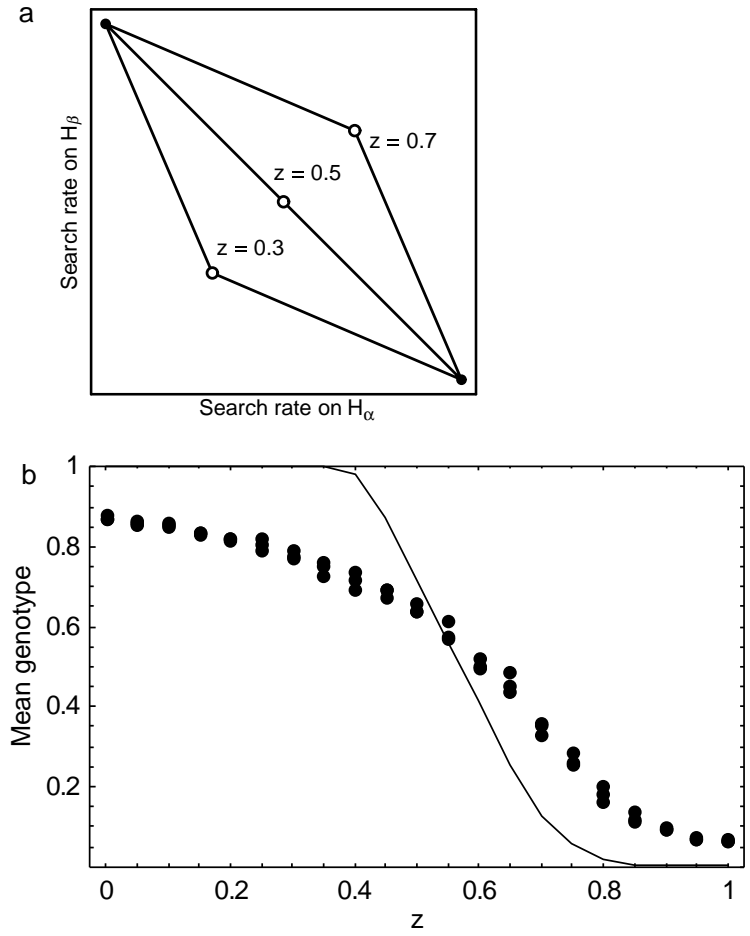

Fig. 4. The tradeoff in search rate for generalist and specialist strategy. (a) The two closed circles are the possible combined search rates for a specialist on host species 1 or 2 . The open circles are the combined search rates for a generalist. Each line represents a different value of $z$. For values of $z>0.5$, generalists are more efficient than specialists. For values of $\mathrm{z}<0.5$, generalists are less efficient than specialists. (b) Evolutionary response of giving-up time to changes in the generalist/specialist tradeoff. The solid line is the equilibrium giving-up time for the deterministic model. Each circle represents a run of the individual-based model. Three simulations are plotted for each value of $\mathrm{z} .(\mathrm{a}=1.16$, $\left.\mathrm{r}_{\alpha}=2.46, \mathrm{r}_{\beta}=1.06, \mathrm{k}_{\alpha}=2.23, \mathrm{k}_{\beta}=0.88\right)$.

compared with specialist parasitoids (z close to 1), or 2) populations cycle. This model makes the prediction that parasitoids with short giving-up times are likely to be found in temporally variable populations. Conversely, specialist parasitoids should more likely evolve in temporally stable populations.

In the presence of endogenously-generated, deterministic cycles, $\mathrm{g}$ can evolve to values less than 0.4. Environmental variation affecting host demographic parameters can also lead to the evolution of reduced giving-up times. The reduction of giving-up times is greater if hosts are affected independently or oppositely by the variable environment. This may be an important mechanism reducing giving-up times given that environmental variation is ubiquitous.

While the empirical literature does not contain enough studies for a thorough comparison of the model results to nature, qualitative differences between empirical results and the model predictions for stable parameters are suggestive. In general, laboratory-based empirical studies have found lower values of $\mathrm{g}$ than predicted by the model. The giving-up times related to odor foraging cues in the literature are near 0.5 or smaller (Table 1). The giving-up time of Cotesia congregata to wild cherry learned at emergence was 6-7 days in a parasitoid with a lifespan of approximately two weeks (Kester and Barbosa 1991). While not specifically examining giving-up time, a study of Leptopilina heterotoma preference for apple-yeast or mushroom, habitats for its Drosophila host, found that the learned preference persisted for less than 4 days, in parasitoids with a lifespan of approximately 11 days (Papaj and Vet 1990). Thus, the limited empirical results are clustered at the bottom of the range of values that the model predicts for stable systems. The presence of endogenous cycling or certain kinds of environmental stochasticity in nature might account for this discrepancy. Within-season temporal variation of host availability has been documented for some of the species in Table 1, but across-season population dynamics remain largely undocumented (Bosque-Pérez et al. 2002, Gonzáles et al. 2002). Clearly other factors could be involved; the most important of these is $\mathrm{z}$, which quantifies the tradeoff in searching rates between generalists and specialist.

A few alternative model formulations have been examined which are mentioned here briefly because of their potential biological relevance and because they illustrate the robust nature of the general conclusions. First, the model presented here makes the simplifying assumption that generalist parasitoids adopt a specialist strategy once they encounter a host. An alternative model was examined in which generalist parasitoids remain generalists for the duration of the season; givingup times typically evolve to even longer times $(>0.5)$, but population variation can still dramatically reduce

Table 1. Giving-up times empirically measured in parasitoid species.

\begin{tabular}{lccc}
\hline & Giving-up time (days) & Lifespan (days) & $\mathrm{g}$ \\
\hline Cotesia Congregata (Kester and Barbosa 1991) & 7 & 14 & 0.5 \\
Leptopilina heterotoma (Papaj and Vet 1990) & 4 & 11 & 0.4 \\
Lysiphlebus testaceipes (Grasswitz and Paine 1993a) & 1 & 3 & 0.3 \\
Diaeretiella rapae (Sheehan and Shelton 1989) & 2 & 6 & 0.3 \\
\hline
\end{tabular}


giving-up times. Second, this model assumes parasitized hosts contribute to density-dependent host regulation (Eq. 3), which is reasonable if parasitism takes place late in the host life cycle or parasitized hosts continue to consume resources. May et al. (1981) argue that the manner in which density dependence is included in the classical model analyzed here (Beddington et al. 1975) represents the minority of real systems, and that other assumptions alter the stability properties of the model. However, limited analysis of a model in which parasitized hosts do not contribute to host densitydependence suggests the general conclusion remains, that cycles reduce the giving-up time.

The model presented here could be further expanded in a number of ways. First, alternative mechanisms for searching or learning may need to be included in order to describe the evolution of giving-up times in parasitoids. Although this model was inspired by experimental studies on the duration of learned foraging odors, the decision-making process of parasitoids may be far more complex, involving a constant tracking and calculation of foraging quality and quantity. For example, dynamic optimization models have been used to successfully model the foraging decisions of the parasitoid Trichogramma thalense on variable quality moths (Keasar et al. 2001) and less successfully to model the evolution of traits in Encarsia formosa (Burger et al. 2004). In addition, parasitoid survival may differ between the host populations. Here I assumed 1 parasitoid was produced from each attacked host, but parasitoid survival can vary between host populations depending on the host (Brodeur et al. 1998) or perhaps the plant on which the host feeds (Salvo and Valladares 2002).

In this model, odor or cue production by plants and hosts is implicitly assumed to be fixed, but induced signals in the plant or even coevolution between induced signals and parasitoid response may be important in understanding natural systems. Learned odors are modeled here as a passive association with the plant-host complex. In contrast, research has demonstrated a link between herbivore induced volatile organic compounds and predator behavior (Kessler and Baldwin 2001). It would be interesting to extend the work presented here to include plant behavior such as induced defenses (1997). Plant behavior and parasitoid behavior may interact in non-intuitive ways. Previous work has demonstrated the sensitivity of the effect of the parasitoid on the specificity of plant info-chemicals in a multi-host system (Vos et al. 2001).

In this study, temporal variation in host populations can have a large effect on the evolution of traits in parasitoids that determine how they search for hosts on which to lay eggs. Low giving-up times are only reachable either in the presence of population cycles or in the presence of non-equal tradeoffs among parasitoid generalists and specialists. While the empirical literature is not extensive enough to distinguish between the possible scenarios described here, the results suggest that one or both of these mechanisms may be important in understanding the evolution of parasitoid giving-up times for cues used to find hosts.

Acknowledgements - I would like to thank Peter A. Abrams, Aneil F. Agrawal, Joyce Besch, Hélène Cyr, Roger M. Nisbet and Brian Shuter for comments on this work. This work was supported in part by an Ontario Graduate Scholarship.

\section{References}

Abrams, P. A. 1997. Evolutionary responses of foragingrelated traits in unstable predator-prey systems. - Evol. Ecol. 11: 673-686.

Abrams, P. A. et al. 1993. On the relationship bewteen quantiative genetic and ESS models. - Evolution 47: $982-985$.

Beddington, J. R. et al. 1975. Dynamic complexity in predator-prey models framed in difference equations. - Nature 255: 58-60.

Bosque-Pérez, N. A. et al. 2002. Species diversity, abundance, and phenology of aphid natural enemies on spring wheats resistant and susceptible to Russian wheat aphid. - Biocontrol 47: 667-684.

Brodeur, J. et al. 1998. Effects of Pieris host species on life history parameters in a solitary specialist and gregarious generalist parasitoid (Cotesia species). - Entomol. Exp. Appl. 86: 145-152.

Burger, J. M. S. et al. 2004. Reproduction now or later: optimal host-handling strategies in the whitefly parasitoid Encarsia formosa. - Oikos 106: 117-130.

Driessen, G. et al. 1995. A count-down mechanism for host search in the parasitoid Venturia canescens. - J. Anim. Ecol. 64: 117-125.

Gillespie, D. T. 1976. General method for numerically simulating the stochastic time evolution of coupled chemical reactions. - J. Comput. Phys. 22: 403-434.

Godfray, H. C. J. 1994. Parasitoids: behavioral and evolutionary ecology. - Princeton, NJ.

Gonzáles, W. L. et al. 2002. Host plant and natural enemy impact on cereal aphid competition in a seasonal environment. - Oikos 96: 481-491.

Grasswitz, T. R. and Paine, T. D. 1993a. Influence of physiological-state and experience on the responsiveness of Lysiphlebus testaceipes (Cresson) (Hymenoptera: Aphidiidae) to aphid honeydew and to host plants. - J. Insect Behav. 6: 511-528.

Grasswitz, T. R. and Paine, T. D. 1993b. Effect of experience on in-flight orientation to host-associated cues in the generalist parasitoid Lysiphlebus testaceipes. - Entomol. Exp. Appl. 68: 219-229.

Green, R. F. 1984. Stopping rules for optimal foragers. - Am. Nat. 123: 30-43.

Griffiths, A. J. F. et al. 1996. An introduction to genetic analysis. - W.H. Freeman. 
Hastings, A. and Godfray, H. C. J. 1999. Learning, host fidelity, and the stability of host-parasitoid communities. - Am. Nat. 153: 295-301.

Iwasa, Y. et al. 1981. Prey distribution as a factor determining the choice of optimal foraging strategy. - Am. Nat. 117: 710-723.

Kaitala, V. et al. 1999. Dynamic complexities in hostparasitoid interaction. - J. Theor. Biol. 197: 331-341.

Keasar, T. et al. 2001. Early oviposition experience affects patch residence time in a foraging parasitoid. - Entomol. Exp. Appl. 98: 123-132.

Kerguelen, V. and Carde, R. T. 1997. Manoeuvres of female Brachymeria intermedia flying towards host-related odours in a wind tunnel. - Physiol. Entomol. 22: 344-356.

Kessler, A. and Baldwin, I. T. 2001. Defensive function of herbivore-induced plant volatile emissions in nature. - Science 291: 2141-2144.

Kester, K. M. and Barbosa, P. 1991. Postemergence learning in the insect parasitoid, Cotesia congregata (Say) (Hymenoptera: braconidae). - J. Insect Behav. 4: 727741 .

May, R. M. et al. 1981. Density dependence in hostparasitoid models. - J. Anim. Ecol. 50: 855-865.

McNair, J. N. 1982. Optimal giving-up times and the marginal value theorem. - Am. Nat. 119: 511-529.

Nuismer, S. L. and Doebeli, M. 2004. Genetic correlations and the coevolutionary dynamics of three-species systems. - Evolution 58: 1165-1177.

Papaj, D. R. and Vet, L. E. M. 1990. Odor learning and foraging success in the parasitoid, Leptopilina heterotoma. - J. Chem. Ecol. 16: 3137-3150.
Salvo, A. and Valladares, G. 2002. Plant-related intraspecific size variation in parasitoids (Hymenoptera: Parasitica) of a polyphagous leafminer (Diptera: Agromyzidae). - Environ. Entomol. 31: 874-879.

Sheehan, W. and Shelton, A. M. 1989. The role of experience in plant foraging by the aphid parasitoid Diaeretiella rapae (Hymenoptera, aphidiidae). - J. Insect Behav. 2: 743759.

Steidle, J. L. M. et al. 2001. The use of general foraging kairomones in a generalist parasitoid. - Oikos 95: 78-86.

Stephens, D. W. and Krebs, J. R. 1986. Foraging theory. - Princeton Univ. Press.

Turlings, T. C. J. et al. 1993. Learning of host-finding cues by Hymenopterous parasitoids. - In: Papaj, D. R. and Lewis, A. C. (eds), Insect learning: ecological and evolutionary perspectives. Chapman \& Hall, pp. 51-78.

Van Alphen, J. J. M. and Galis, F. 1983. Patch time allocation and parasitization efficiency of Asobara tabida, a larval parasitoid of Drosophila. - J. Anim. Ecol. 52: 937-952.

Vet, L. E. M. and Groenewold, A. W. 1990. Semiochemicals and learning in parasitoids. - J. Chem. Ecol. 16: 31193136.

Vos, M. et al. 2001. Plant-mediated indirect effects and the persistence of parasitoid-herbivore communities. - Ecol. Lett. 4: 38-45.

Waage, J. K. 1979. Foraging for patchily-distributed hosts by the parasitoid, Nemeritis canescens. - J. Anim. Ecol. 48: 353-371. 